\title{
Some Wave Functions and Potential Functions Pertaining to Spherically Stratified Media ${ }^{1}$
}

\author{
C. T. Tai \\ Contribution from Antenna Laboratory, Department of Electrical Engineering, Ohio State University, \\ Columbus, Ohio
}

(Received September 20, 1962)

\begin{abstract}
The wave functions pertaining to a bilinearly stratified medium are presented in this paper. Solutions to the equation $\nabla \cdot[\kappa(r) \nabla \psi]=0$ have been investigated for several profiles of $\kappa(r)$. An analysis is given to the equation $\nabla \cdot\left[\kappa^{\circ} \cdot \nabla \psi\right]=-\rho / \epsilon_{0}$ which arises from the formulation of the quasi-static electric field in a homogeneous anisotropic medium.
\end{abstract}

\section{Introduction}

It is known that the core of all electromagnetic problems involving a spherically stratified medium consists of two second order linear differential equations of the form:

$$
\frac{d^{2} S_{n}}{d r^{2}}+\left[k^{2} \kappa(r)-\frac{n(n+1)}{r^{2}}\right] S_{n}=0
$$

and

$$
\kappa(r) \frac{d}{d r}\left[\frac{1}{\kappa(r)} \frac{d T_{n}}{d r}\right]+\left[k^{2} \kappa(r)-\frac{n(n+1)}{r^{2}}\right] T_{n}=0
$$

where

$$
\begin{aligned}
k^{2} & =\omega^{2} \mu_{0} \epsilon_{0}, \\
\kappa(r) & =\frac{\epsilon(r)}{\epsilon_{0}} .
\end{aligned}
$$

Based upon the two sets of scalar functions $S_{n}$ and $T_{n}$, four sets of vector wave functions may be constructed to describe the electromagnetic field within such a medium. The formal solutions to the spherical Luneberg lens [Tai, 1958], the cylindrica] Luneberg lens [Tai, 1956], and the Maxwell fish eyes [Tai, 1958], have already been reported based upon this method. We shall present here the solutions to (1) and (2) corresponding to a bilinear distribution of $\kappa(r)$ that contains the stratification of the conical lens of Luneberg [1944] as a special case.

In studying the scattering of electromagnetic waves by small spherically stratified particles, it is not necessary to find the complete wave solution. By means of the Rayleigh method, or its extension [Tai, 1952], it is sufficient to investigate the solution to the potential function $\psi$ which satisfies the differential equation

1 The research reported here was performed under Contract AF 33(616)-6782 sponsored by Aeronautical Systems Division, Wright-Patterson Air Force Base, with the Ohio State University Research Foundation. The part of this paper on the wave functions was presented orally at the URSI Meeting in paper on the wave fun
Washington, May 1961.

$$
\nabla \cdot[\kappa(r) \nabla \psi]=0 .
$$

We shall summarize here those cases in which the wave functions have already been found for the sume profile of $\kappa(r)$.

Finally, a brief discussion will be given to the solution of the equation

$$
\nabla \cdot[\dot{\kappa} \cdot \nabla \psi]=-\rho / \epsilon_{0},
$$

where $\overleftrightarrow{\kappa}$ denotes a homogeneous dielectric tensor. Equation (4) arises from a consideration of the quasi-static solution to the electric field in an anisotropic medium.

\section{Wave Functions Pertaining to a Bilinearly Stratified Medium}

A bilinearly stratified medium is defined as one with the dielectric "constant" varying according to the following relation:

$$
\kappa(r)=\frac{\epsilon(r)}{\epsilon_{0}}=K_{\infty}\left(\frac{r+r_{1}}{r+r_{2}}\right) .
$$

By writing

$$
r_{1}=\frac{K_{0}}{K_{\infty}} r_{2}
$$

(5) becomes

$$
\kappa(r)=K_{\infty}\left[\frac{r+\left(\frac{K_{0}}{K_{\infty}}\right) r_{2}}{r+r_{2}}\right],
$$

where $K_{0}$ and $K_{\infty}$ denote, respectively, the values of $\kappa(r)$ defined at $r=0$ and $r=\infty$. At $r=r_{2}$, the value of $\kappa\left(r_{2}\right)$ is equal to the mean of $K_{0}$ and $K_{\infty}$. One may, therefore, choose the proper values of $K_{0}, K_{\infty}$, and $r_{2}$ to simulate a monotonically increasing or decreasing function of $\kappa(r)$ which has a finite asymptotic value at $r=\infty$. 
For convenience, the independent variable $r$ in (1) and (2) will be changed to $\rho$ by letting $\rho=k r$, then (5) becomes

$$
\kappa(\rho)=K_{\infty}\left(\frac{\rho+\rho_{1}}{\rho+\rho_{2}}\right)
$$

where $\rho_{1}=k r$, and $\rho_{2}=k r_{2}$. By substituting (7) into (1), and letting

$$
S_{n}=\left(\rho+\rho_{2}\right) \rho^{n+1} U_{n},
$$

one finds that the function $U_{n}$ satisfies the following differential equation:

$$
U_{n}^{\prime \prime}+\left(\frac{2 n+1}{\rho}+\frac{2}{\rho+\rho_{2}}\right) U_{n}^{\prime}+\left(a+\frac{a_{0}}{\rho}+\frac{a_{1}}{\rho+\rho_{2}}\right) U_{n}=0
$$

where

$$
\begin{aligned}
a & =K_{\infty}, a_{0}=\frac{2 n+1}{\rho_{2}}, \\
a_{1} & =K_{\infty}\left(\rho_{1}-\rho_{2}\right)-\frac{2 n+1}{\rho_{2}},
\end{aligned}
$$

and the prime on $U_{n}$ in (9) denotes the derivative with respect to $\rho$. The function $U_{n}$ has two regular singularities and one irregular singularity at infinity of the second species. Adopting the notation of Ince [1944], one would designate this by $\left[0,2,1_{2}\right]$. The same type of function occurs in the theory of the spherical Luneberg lens [Tai, 1958]. However, in that case it was the $T_{n}$ function which is of this type. The $S_{n}$ function in the theory of the Luneberg lens is expressible in terms of the confluent hypergeometric function.

By substituting (7) into (2) and letting

$$
T_{n}=\rho^{n+1} V_{n},
$$

one finds that $V_{n}$ satisfies the following equation:

$$
\begin{aligned}
V_{n}^{\prime \prime}+\left(\frac{2 n+1}{\rho}-\right. & \left.\frac{1}{\rho+\rho_{1}}+\frac{1}{\rho+\rho_{2}}\right) V_{n}^{\prime} \\
& +\left(b+\frac{b_{0}}{\rho}+\frac{b_{1}}{\rho+\rho_{1}}+\frac{b_{2}}{\rho+\rho_{2}}\right) V_{n}=0,
\end{aligned}
$$

where

$$
\begin{aligned}
b & =K_{\infty}, b_{0}=(2 n+1)\left(\frac{1}{\rho_{2}}-\frac{1}{\rho_{1}}\right), \\
b_{1} & =\frac{2 n+1}{\rho_{1}}, b_{2}=K_{\infty}\left(\rho_{1}-\rho_{2}\right)-\frac{2 n+1}{\rho_{2}} .
\end{aligned}
$$

The function $V_{n}$ has one more regular singular at $\rho=-\rho_{2} . \quad$ It is of the type $\left[0,3,1_{2}\right]$.

When one puts $r_{2}=0$ in $(7)$, the distribution of $\kappa(r)$ becomes the same as the profile of the conical lens of Luneberg [1944j. The $U_{n}$ and $V_{n}$ functions then reduce, respectively, to the types $\left[0,1,1_{2}\right]$ and $\left[0,2,1_{2}\right]$. The type $\left[0,1,1_{2}\right]$ is simply the confluent hypergeo- metric function. It is interesting to observe that the same two types of functions are involved in the electromagnetic theory of the spherical Luneberg lens.

\section{Some Potential Functions}

In either purely electrostatic problems dealing with a spherically stratified medium or scattering problems involving small spherical particles, one would encounter the equation

$$
\nabla \cdot[\kappa(r) \nabla \psi]=0
$$

By applying the method of separating variables in a spherical coordinate system, one finds that $\psi$ can be expressed in terms of the following characteristic functions, i.e.,

$$
\psi=W_{n}(r) P_{n}^{m}(\cos \theta)_{\sin }^{\cos } m \phi,
$$

where $W_{n}(r)$ satisfies the equation

$$
\frac{d}{d r}\left[r^{2} \kappa(r) \frac{d W_{n}}{d r}\right]-n(n+1) \kappa(r) W_{n}=0,
$$

and $P_{n}^{m}(\cos \theta)$ denotes the associated Legendre functions. The solutions to (14) for several profiles of $\kappa(r)$ are given below.

\subsection{Luneberg Lens: $\kappa(r)=2-r^{2}$}

In this case the radial function $W_{n}(r)$ is expressible in terms of the hypergeometric function. The function which is regular at $r=0$ is given by

$$
W_{n}=\left(\frac{r^{2}}{2}\right)^{\frac{n}{2}} F\left(a, b, c ; \frac{r^{2}}{2}\right)
$$

where $F(a, b, c ; z)$ denotes the hypergeometric function, and

$$
\begin{aligned}
a & =\frac{n}{2}+\frac{3}{4}+\frac{1}{2}\left[\left(n+\frac{1}{2}\right)^{2}+2\right]^{1 / 2} \\
b & =\frac{n}{2}+\frac{3}{4}-\frac{1}{2}\left[\left(n+\frac{1}{2}\right)^{2}+2\right]^{1 / 2} \\
c & =n+\frac{3}{2} .
\end{aligned}
$$

3.2. Maxwell Fisheyes: $\kappa(r)=\frac{4}{\left(1+r^{2}\right)^{2}}$.

The radial function, again, is expressible in terms of the hypergeometric function. The function which is regular at $r=0$ is

$$
W_{n}(r)=\left(-r^{2}\right)^{\frac{n}{2}} F\left(a, b, c ;-r^{2}\right),
$$


where

$$
\begin{aligned}
& a=\frac{n}{2}-\frac{3}{4}+\frac{1}{2}\left[\left(n+\frac{1}{2}\right)^{2}+2\right]^{\frac{1}{2}} \\
& b=\frac{n}{2}-\frac{3}{4}-\frac{1}{2}\left[\left(n+\frac{1}{2}\right)^{2}+2\right]^{\frac{1}{2}} \\
& c=n+\frac{3}{2} .
\end{aligned}
$$

\subsection{Bilinear Distribution: $\kappa(r)=K_{\infty} \frac{r+r_{1}}{r+r_{2}}$}

By letting $W_{n}=r^{n} Z_{n}$, one finds that $Z_{n}$ satisfies the equation

$Z_{n}^{\prime \prime}+\left[\frac{2(n+1)}{r}+\frac{1}{r+r_{1}}+\frac{1}{r+r_{2}}\right] Z_{n}^{\prime}$

$$
+\frac{n}{r}\left(\frac{1}{r+r_{1}}-\frac{1}{r+r_{2}}\right) Z_{n}=0 .
$$

In contrast to the wave functions for the same profile of $\kappa(r)$, it can be verified that the function $Z_{n}(r)$ has a regular singularity at infinity, hence it is of the type $[0,4,0]$. The function is, therefore, similar to the hypergeometric function which is designated by $[0,3,0]$. It is obvious from (17) that when $r_{2}=0$, corresponding to the conical lens distribution of Luneberg, $Z_{n}$ reduces to the hypergeometric function.

\section{Equation $\nabla \cdot[\stackrel{\leftrightarrow}{\kappa} \cdot \nabla \psi]=-\rho / \epsilon_{0}$ and Its Solution}

The foundation of the electromagnetic theory dealing with a harmonically varying field in a magnetoionic medium is based upon the following Maxwell equations:

$$
\begin{gathered}
\nabla \times \vec{E}=-j \mu_{0} \omega \vec{H}, \\
\nabla \times \vec{H}=\vec{J}+j \omega \epsilon_{0} \kappa \cdot \vec{E},
\end{gathered}
$$

where $\vec{J}$ denotes the current density function not of magneto-ionic origin. The dielectric tensor $\kappa$, for the case that the static magnetic field is pointed in the $z$-direction, is given by

$$
\overleftrightarrow{\kappa}=\left[\begin{array}{ccc}
\kappa_{11} & \kappa_{12} & 0 \\
-\kappa_{12} & \kappa_{11} & 0 \\
0 & 0 & \kappa_{33}
\end{array}\right] \text {. }
$$

Numerous authors have recently investigated the complete wave solutions resulting from (18) and (19). We would like to mention particularly the works of Bunkin [1957] and Arbel [1960], where both use the Fourier-transform method in deriving the formal solution to the problem. The resultant integrals can be evaluated by the asymptotic method to yield the far-zone field of a radiating element No explicit solution is vet available for the nearzone field except the brief description given by Mittra and Deschamps [1962] in the Abstract of the recent Copenhagen Electromagnetic Symposium (July 1962). The interesting problem of the scattering of an electromagnetic wave by an obstacle in an anisotropic medium is yet to be solved. The problem of the scattering of a plane wave by a small spherical anisotropic body in air has successfully been solved by Berk and Lengyl [1955]. With this brief introduction it seems of some interest to uncover whatever information we can gather from (18) and (19), even in a restricted form. We shall consider here the quasi-static solution to the electric field by neglecting the right term of (18), and hence start with

$$
\nabla \times \vec{E}=0
$$

and the divergence equation resulting from (19) and the equation of continuity, i.e.,

$$
\nabla \cdot\left[{ }^{\prime} \kappa \cdot \vec{E}\right]=\frac{\rho}{\epsilon_{0}} .
$$

In view of (21), one may introduce a potential function $\psi$ such that

$$
\vec{E}=-\nabla \psi
$$

Substituting (23) into (22) one obtains

$$
\nabla \cdot[\stackrel{\Delta}{\wedge} \cdot \nabla \psi]=-\frac{\rho}{\epsilon_{0}} .
$$

In a cartesian coordinate system, (24) can be written as

$$
\kappa_{11}\left(\frac{\partial^{2} \psi}{\partial x^{2}}+\frac{\partial^{2} \psi}{\partial y^{2}}\right)+\kappa_{33} \frac{\partial^{2} \psi}{\partial z^{2}}=-\frac{\rho}{\epsilon_{0}},
$$

where we have already made use of (20). To facilitate the analysis, we shall first consider the case where $\rho$ represents that of a single charge located at the origin; i.e., we let

$$
\rho=q \delta(\vec{r}-0),
$$

where $\delta(\vec{r}-0)$ denotes the three-dimensional delta function. Equation (25) can then be written as

$$
\frac{\partial^{2} \psi}{\partial x^{2}}+\frac{\partial^{2} \psi}{\partial y^{2}}+\alpha^{2} \frac{\partial^{2} \psi}{\partial z^{2}}=-\frac{q}{\epsilon_{0} \kappa_{11}} \delta(\vec{r}-0)
$$

By means of the Fourier-transform method or by transforming (27) into the standard form of Poisson's equation by letting $x^{\prime}=\alpha x$ and $y^{\prime}=\alpha y$, one finds that the solution to (27) is given by

$$
\psi=\frac{q}{4 \pi \epsilon_{0} \kappa_{11} R_{\alpha}},
$$


where

$$
R_{\alpha}=\left[\alpha^{2}\left(x^{2}+y^{2}\right)+z^{2}\right]^{\frac{1}{2}} .
$$

Based upon this elementary solution, the field of a dipole situated at the origin and of moment $p$ pointed in the z-direction can be derived. The result is given by

where

$$
\psi_{p}=\frac{\vec{p} \cdot \vec{R}}{4 \pi \epsilon_{0} \kappa_{11} R_{\alpha}^{3}}
$$

$$
R=\sqrt{x^{2}+y^{2}+z^{2}} .
$$

The corresponding electric field can be found by means of (23). The dependence of $\vec{E}$ upon the modified radial distance $R_{\alpha}$ concurs with the principal term of the near-zone field of an oscillating dipole in a magneto-ionic medium as obtained by Mittra and Deschamps through the wave solution. Equation (28) may also be used to find the characteristic functions pertaining to the homogeneous equation

$$
\frac{\partial^{2} \psi}{\partial x^{2}}+\frac{\partial^{2} \psi}{\partial y^{2}}+\alpha^{2} \frac{\partial^{2} \psi}{\partial z^{2}}=0
$$

through the technique of multipole expansion. It is, however, more convenient to find these solutions by changing (30) into the standard form of the Laplace equation

where

$$
\frac{\partial^{2} \psi}{\partial x_{\alpha}^{2}}+\frac{\partial^{2} \psi}{\partial y_{\alpha}^{2}}+\frac{\partial^{2} \psi}{\partial z^{2}}=0
$$

$$
x_{\alpha}=\alpha x, y_{\alpha}=\alpha y .
$$

The characteristic functions, when expressed in a spherical coordinate system, are then given by

$$
\psi_{n m_{0}^{e}}=\left[A_{n} R_{\alpha}^{n}+B_{n} R_{\alpha}^{-(n+1)}\right] P_{n}^{m}\left(\cos \theta_{\alpha}\right)_{\sin }^{\cos } m \phi,
$$

where

$$
\begin{aligned}
R_{\alpha} & =\left[\alpha^{2}\left(x^{2}+y^{2}\right)+z^{2}\right]^{1 / 2} \\
& =R\left[\alpha^{2}+\left(1-\alpha^{2}\right) \cos ^{2} \theta\right]^{1 / 2} \\
\cos \theta_{\alpha} & =\frac{z}{R_{\alpha}}=\frac{\cos \theta}{\left[\alpha^{2}+\left(1-\alpha^{2}\right) \cos ^{2} \theta\right]^{1 / 2}} .
\end{aligned}
$$

The dipole field as given by (29) corresponds to the function

$$
B_{1} R_{\alpha}^{-2} P_{1}\left(\cos \theta_{\alpha}\right) \text {. }
$$

In the work of Berk and Lengyl [1955], only the characteristic functions

$$
R_{\alpha} P_{1}^{t}\left(\cos \theta_{\alpha}\right)_{\sin }^{\cos } \phi=\alpha R P_{1}^{t}(\cos \theta)_{\sin }^{\cos } \phi=\alpha\left\{\begin{array}{l}
x \\
y
\end{array}\right.
$$

are needed to satisfy the required boundary conditions for the corresponding magnetostatic problem. There seems to be no simple way to find the solution for the complementary problem of a small spherical dielectric body placed in an anisotropic medium. The only problem of this nature that can be solved neatly consists of a perfectly conducting spheroıd with a contour defined by

$$
\alpha^{2}\left(x^{2}+y^{2}\right)+z^{2}=K^{2} .
$$

When such a spheroid is placed in an anisotropic medium, then an incident field defined by

$$
\psi_{i}=-E_{0} x
$$

would produce a scattered field given by

$$
\psi_{s}=E_{0} \frac{K^{3}}{R_{\alpha}^{2}} P_{1}^{\prime}\left(\cos \theta_{\alpha}\right) \cos \phi .
$$

The author gratefully acknowledges the discussions which he had with Dr. H. Unz on the problem involving an anisotropic medium.

\section{References}

Arbel, E. (1960), Radiation from a point source in an anisotropic medium, Res. Rept. PIB-MRI-861-60, Polytech. Inst. Brooklyn, N.Y.

Berk, A. D., and B. A. Lengyl (1955), Magnetic fields in small ferrite bodies with applications to microwave cavities containing such bodies, Proc. IRE 43, 1587-1591.

Bunkin, F. V. (1957), On the radiation in anisotropic medium, J.E.T.P. (USSR) 32, 338-346.

Ince, E. L. (1944), Ordinary differential equations (Dover Publications, New York)

Luneberg, R. K. (1944), The mathematical theory of optics (Brown University Press, Providence, R.I.).

Mittra, R., and G. A. Deschamps (1962), Field solution for a dipole in an anisotropic medium, Symposium on Electromagnetic Theory and Antennas, Copenhagen.

Tai, C. T. (1952), Quasi-static solution for diffraction of a plane electromagnetic wave by a small spheroid, IRE Trans. Antennas Propagation PGAP-1, 13-26.

Tai, C. T. (1956), Theory of the cylindrical Luneberg lens excited by a line magnetic current, Report No. 678-3, Antenna Laboratory, Department of Electrical Engineering, Ohio State University.

Tai, C. T. (1958), Maxwell fish-eye treated by Maxwell equations, Nature 182, 1600-1601.

Tai, C. T. (1958), The electromagnetic theory of the spherical Luneberg lens, Appl. Sci. Res. Sect. B \%, 113-130. 